\title{
A Symplectic Conservative Perturbation Series Expansion Method for Linear Hamiltonian Systems with Perturbations and Its Applications
}

\author{
Zhiping Qiu* and Nan Jiang \\ Institute of Solid Mechanics, School of Aeronautic Science and Engineering, Beihang \\ University, Beijing 100083, China
}

Received 1 September 2020; Accepted (in revised version) 24 December 2020

\begin{abstract}
In this paper, a novel symplectic conservative perturbation series expansion method is proposed to investigate the dynamic response of linear Hamiltonian systems accounting for perturbations, which mainly originate from parameters dispersions and measurement errors. Taking the perturbations into account, the perturbed system is regarded as a modification of the nominal system. By combining the perturbation series expansion method with the deterministic linear Hamiltonian system, the solution to the perturbed system is expressed in the form of asymptotic series by introducing a small parameter and a series of Hamiltonian canonical equations to predict the dynamic response are derived. Eventually, the response of the perturbed system can be obtained successfully by solving these Hamiltonian canonical equations using the symplectic difference schemes. The symplectic conservation of the proposed method is demonstrated mathematically indicating that the proposed method can preserve the characteristic property of the system. The performance of the proposed method is evaluated by three examples compared with the Runge-Kutta algorithm. Numerical examples illustrate the superiority of the proposed method in accuracy and stability, especially symplectic conservation for solving linear Hamiltonian systems with perturbations and the applicability in structural dynamic response estimation.
\end{abstract}

AMS subject classifications: 74H15, 37N15, 65P10, 65M06

Key words: Linear Hamiltonian system, perturbation series expansion method, symplectic structure, symplectic algorithm, structural dynamic response.

\section{Introduction}

With the dramatically rapid development of science and technology, numerical calculation has aroused more and more attention, thus there is an urgent need for more efficient

*Corresponding author.

Email: zpqiu@buaa.edu.cn (Z. Qiu) 
and more stable numerical algorithms with more powerful long-term simulation capabilities.

From the perspective of mechanical systems, the conservative Newton's equation can be represented to two equivalent mathematical forms: a Lagrangian variation form and a Hamiltonian form. Compared to other forms, the Hamiltonian formulation have a symmetric form, and any physical process with negligible dissipations can be expressed in the form of a Hamiltonian system [1]. The equivalent representations describe the same physics law but provide different techniques in solving the same problem, thus may produce different numerical results [2]. Therefore, making reasonable and sensible choices from various equivalent representations are crucial.

Numerical algorithms should preserve the intrinsic properties of the original problems as much as possible [3-5]. It is worth noting that apart from some very rare exceptions, almost all the conventional algorithms are non-symplectic [6], which may lead to serious distortions of numerical results. They can be used in short-term simulation, but may result in wrong conclusions for long-term tracking research [7]. On the contrary, the symplectic algorithms of Hamiltonian systems can avoid all non-symplectic pollution and conserve the symplectic structure of the system, which is the characteristic property of the Hamiltonian system [8]. Therefore, the symplectic algorithms have significant advantages in long-term numerical simulation $[9,10]$.

Pioneering work on symplectic algorithms is due to Feng [11], who first proposed in 1984 at the international conference on differential geometry and equations. His work represented a milestone in the development of numerical calculation [12] and attracted extensive attention from scholars at home and abroad. Subsequently, lots of researchers obtained many major results on symplectic algorithms. The judging conditions of symplectic Runge-Kutta methods was found in 1988 by Sanz-Serna [13], Lasagni [14] and Suris [15] independently. Then, Sun [16] studied symplectic partitioned Runge-Kutta methods deeply. Around 2000, Bridges [17] and Reich [18] first put forward the multisymplectic algorithms. In recent years, the meshless symplectic algorithms $[19,20]$, the symplectic continuous-stage Runge-Kutta methods [21,22], the Fourier spectral/ pseudospectral methods $[23,24]$, and other symplectic algorithms have been developed in succession. A large number of numerical simulations indicate that the symplectic algorithms have superiority in conservation and long-term tracking ability.

In terms of the symplectic algorithms applied in structural dynamic response analysis, Zhang [25] proposed a symplectic algorithm for the dynamic response of the Timoshenko beam. Hu [26] utilized a multi-symplectic method to analyze the dynamic response of the multi-span continuous beam. Li [27] put forward a symplectic method for the dynamic response of the harmonic oscillator and simply supported beam. Yang [28] performed the numerical simulations of the super slender Kirchhoff rod by the symplectic algorithm. Xing [29] developed two highly precise symplectic schemes for linear structural dynamic analysis. Zhang [30] applied the symplectic Runge-Kutta method in the dynamics of spacecraft relative motion. Peng [31,32] proposed the symplectic nonsmooth dynamic method for multibody system analysis. The numerical results all illus- 
trated high accuracy and stability as well as good conservation properties of the symplectic algorithms above.

In reality, no one can deny the fact that errors, or what we call perturbations, exist inevitably in practical engineering applications, where materials dispersions and measurement deviations are the main roots [33-37]. Even small perturbations may result in a significant reduction of structural performance, even structural failure [38-41]. The perturbation theory $[42,43]$ provides a workable approach for performing perturbation analysis to ensure the safety of structures. It involves the use of an artificial small parameter for numerical analysis [44], from which a set of recursive equations can be derived and then the approximate solution to the response can be obtained. The perturbation series solution is considered as a modification of the nominal solution obtained by the deterministic method. Due to its conspicuous features of high computational efficiency and good approximation accuracy [45], the perturbation method has been proven to be a valid and efficient approach in various fields, including structural dynamic response calculation [46], structural eigenvalue problems [47], fatigue crack growth evolution predicting $[48,49]$ and so on. Recently, Qiu [50,51] has applied the perturbation theory to analyze the dynamic response of linear Hamiltonian systems and Birkhoffian systems with stochastic and interval uncertainties, respectively. Despite above valuable contributions, to the authors' best knowledge, there has been very little research taking advantage of the perturbation method to investigate the effects of deterministic perturbations on the dynamic response of Hamiltonian systems in the literature.

Motivated by the previous work, the main contribution of this paper is to put forward a symplectic conservative perturbation series expansion method for linear Hamiltonian systems with consideration of perturbations. In this respect, the linear Hamiltonian system with perturbations is transformed into a series of Hamiltonian canonical equations by introducing a small parameter, which can be solved by the symplectic difference schemes. Thereby the time response of the Hamiltonian system can be obtained successfully. The proposed method can give highly accurate results in dynamic response evaluation. Most importantly, it can conserve the characteristics of the system showing its unique superiority in symplectic conservation.

The remainder of this paper is structured as follows. The linear Hamiltonian system and its symplectic structure are introduced in Section 2. In Section 3, the perturbation series expansion method is proposed for the linear Hamiltonian system with perturbations and its symplecticity is demonstrated. Then, the symplectic difference schemes for Hamiltonian systems are presented to solve the Hamiltonian canonical equations in Section 4 . Three examples are accomplished to illustrate the validity, symplectic conservation and engineering applicability of the proposed method in Section 5, and Section 6 presents a brief conclusion at last.

\section{Linear Hamiltonian system and its symplectic structure}




\subsection{Linear Hamiltonian system}

Consider the Hamiltonian canonical equation in a compact form

$$
\frac{d \mathbf{z}}{d t}=\mathbf{J}^{-1} H_{\mathbf{z}}
$$

where

$$
\mathbf{z}=\left(z_{1}, \cdots, z_{n}, z_{n+1}, \cdots, z_{2 n}\right)^{T}, \quad H_{\mathbf{z}}=\left(\frac{\partial H}{\partial z_{1}}, \cdots, \frac{\partial H}{\partial z_{2 n}}\right)^{T}, \quad \mathbf{J}=\left(\begin{array}{cc}
\mathbf{0} & \mathbf{I}_{n} \\
-\mathbf{I}_{n} & \mathbf{0}
\end{array}\right),
$$

in which $\mathbf{I}_{n}$ is the $n \times n$ identity matrix; $\mathbf{J}$ is called the standard symplectic matrix, which has the property $\mathbf{J}^{-1}=\mathbf{J}^{T}=-\mathbf{J} ; H$ is called the Hamiltonian function of the system.

A Hamiltonian system (2.1) is called linear, if the Hamiltonian function $H(\mathbf{z})$ is a quadratic form of $\mathbf{z}$

$$
H(\mathbf{z})=\frac{1}{2} \mathbf{z}^{T} \mathbf{C} \mathbf{z},
$$

where $\mathbf{C}$ is a symmetric matrix, namely, $\mathbf{C}^{T}=\mathbf{C}$.

Thus, the Hamiltonian canonical equation (2.1) can be expressed as

$$
\frac{d \mathbf{z}}{d t}=\mathbf{B z},
$$

where $\mathbf{B}=\mathbf{J}^{-1} \mathbf{C}$ is infinitesimal symplectic, which is also called the Hamiltonian operator matrix in the symplectic space.

\subsection{Symplectic structure of the linear Hamiltonian system}

Symplecticity is the essential characteristic of a Hamiltonian system. The phase space of the Hamiltonian system is equipped with a standard symplectic structure written as a closed differential 2-form [2]

$$
\omega=\sum_{i=1}^{n} d z_{i} \wedge d z_{n+i},
$$

where the notation $\wedge$ denotes the exterior product.

Based on the property of the exterior product, Eq. (2.5) can be transformed into the following expression

$$
\begin{aligned}
\omega & =\frac{1}{2} \times\left(2 \sum_{i=1}^{n} d z_{i} \wedge d z_{n+i}\right)=\frac{1}{2} \times\left[\sum_{i=1}^{n} d z_{i} \wedge d z_{n+i}+\sum_{i=1}^{n} d z_{n+i} \wedge\left(-d z_{i}\right)\right] \\
& =\frac{1}{2} \times\left(d z_{1}, \cdots, d z_{n}, d z_{n+1}, \cdots, d z_{2 n}\right)^{T} \wedge\left(d z_{n+1}, \cdots, d z_{2 n},-d z_{1}, \cdots,-d z_{n}\right)^{T} \\
& =\frac{1}{2} d \mathbf{z} \wedge \mathbf{J} d \mathbf{z} .
\end{aligned}
$$


Let

$$
\mathbf{z}_{t}=\frac{d \mathbf{z}}{d t}
$$

The differential form of the symplectic structure $\omega$ is

$$
\frac{d \omega}{d t}=\frac{d}{d t}\left(\frac{1}{2} d \mathbf{z} \wedge \mathbf{J} d \mathbf{z}\right)=\frac{1}{2}\left(d \mathbf{z}_{t} \wedge \mathbf{J} d \mathbf{z}+d \mathbf{z} \wedge \mathbf{J} d \mathbf{z}_{t}\right)=d \mathbf{z} \wedge \mathbf{J} d \mathbf{z}_{t}
$$

The variational equation of the Hamiltonian canonical equation (2.1) is

$$
\mathbf{J} d \mathbf{z}_{t}=H_{\mathbf{z z}}(\mathbf{z}) d \mathbf{z},
$$

where $H_{\mathbf{z z}}(\mathbf{z})$ is the Hessian matrix of the function $H(\mathbf{z})$.

Taking the exterior product of Eq. (2.9) with $d \mathbf{z}$ leads to

$$
d \mathbf{z} \wedge \mathbf{J} d \mathbf{z}_{t}=d \mathbf{z} \wedge H_{\mathbf{z z}}(\mathbf{z}) d \mathbf{z} .
$$

It is worth noting that since $H_{\mathrm{zz}}$ is symmetric, the right term of Eq. (2.10) become

$$
d \mathbf{z} \wedge H_{\mathbf{z z}}(\mathbf{z}) d \mathbf{z}=-H_{\mathbf{z z}}(\mathbf{z}) d \mathbf{z} \wedge d \mathbf{z}=H_{\mathbf{z z}}(\mathbf{z}) d \mathbf{z} \wedge d \mathbf{z}=0 .
$$

Therefore, along the phase flow, it holds the symplecticity, i.e., [52]

$$
\frac{d \omega}{d t}=d \mathbf{z} \wedge \mathbf{J} d \mathbf{z}_{t}=0
$$

\section{Perturbation series expansion method of the linear Hamiltonian system with perturbations and its symplectic structure}

\subsection{Perturbation series expansion method of the linear Hamiltonian system with perturbations}

Traditionally, the dynamic response of the linear Hamiltonian system can be obtained by solving Eq. (2.4) directly. However, as we discussed above, the system is generally affected by some disturbances and perturbations. When there are perturbations in the linear Hamiltonian system, the perturbation theory, which studies the behavior changes of a system subjected to small changes in its variables [43], can be applied to obtain the approximate solution. By introducing a small parameter, the infinitesimal symplectic matrix $\mathbf{B}$ can be expressed as a sum of the nominal value and the perturbation, that is

$$
\mathbf{B}=\mathbf{B}_{0}+\varepsilon \mathbf{B}_{r}
$$


where the small parameter $\varepsilon$ is a scalar quantity much less than unity; $\mathbf{B}_{0}$ denotes the nominal part of $\mathbf{B}, \varepsilon \mathbf{B}_{r}$ denotes the perturbation of $\mathbf{B}_{0}, \mathbf{B}_{0}$ and $\mathbf{B}_{r}$ are both infinitesimal symplectic.

On this account, there exist consequent perturbations in $\mathbf{z}$. Based on the perturbation theory, $\mathbf{z}$ can be expanded as the form of power series with $\varepsilon$

$$
\mathbf{z}=\mathbf{z}_{0}+\varepsilon \mathbf{z}_{1}+\varepsilon^{2} \mathbf{z}_{2}+\cdots+\varepsilon^{m} \mathbf{z}_{m}+\cdots,
$$

where $\mathbf{z}_{0}$ is the nominal part of $\mathbf{z}, m$ denotes a positive integer and $\mathbf{z}_{i},(i=1, \cdots, m)$ is the $i$ th-order perturbation of $\mathbf{z}$.

Substituting Eqs. (3.1) and (3.2) into Eq. (2.4) yields

$$
\begin{aligned}
& \frac{d\left(\mathbf{z}_{0}+\varepsilon \mathbf{z}_{1}+\varepsilon^{2} \mathbf{z}_{2}+\cdots+\varepsilon^{m} \mathbf{z}_{m}+\cdots\right)}{d t} \\
= & \left(\mathbf{B}_{0}+\varepsilon \mathbf{B}_{r}\right)\left(\mathbf{z}_{0}+\varepsilon \mathbf{z}_{1}+\varepsilon^{2} \mathbf{z}_{2}+\cdots+\varepsilon^{m} \mathbf{z}_{m}+\cdots\right) .
\end{aligned}
$$

Expanding Eq. (3.3), then comparing the coefficients of the same power terms of $\varepsilon$ on each side of the equation, we can obtain a series of Hamiltonian canonical equations

$$
\begin{aligned}
& \varepsilon^{0}: \frac{d \mathbf{z}_{0}}{d t}=\mathbf{B}_{0} \mathbf{z}_{0}, \\
& \varepsilon^{1}: \frac{d \mathbf{z}_{1}}{d t}=\mathbf{B}_{0} \mathbf{z}_{1}+\mathbf{B}_{r} \mathbf{z}_{0}, \\
& \varepsilon^{2}: \frac{d \mathbf{z}_{2}}{d t}=\mathbf{B}_{0} \mathbf{z}_{2}+\mathbf{B}_{r} \mathbf{z}_{1}, \cdots, \\
& \varepsilon^{m}: \frac{d \mathbf{z}_{m}}{d t}=\mathbf{B}_{0} \mathbf{z}_{m}+\mathbf{B}_{r} \mathbf{z}_{m-1} .
\end{aligned}
$$

In this way, the Hamiltonian functions $H_{i},(i=0,1, \cdots, m)$ and their derivatives $H_{\mathbf{z}_{i}}$, $(i=$ $0,1, \cdots, m)$ of the Hamiltonian canonical equations in Eq. (3.4) are presented respectively as

$$
\begin{array}{ll}
H_{0}\left(\mathbf{z}_{0}\right)=\frac{1}{2} \mathbf{z}_{0}^{T} \mathbf{C}_{0} \mathbf{z}_{0}, & H_{\mathbf{z}_{0}}=\mathbf{C}_{0} \mathbf{z}_{0}, \\
H_{1}\left(\mathbf{z}_{1}\right)=\frac{1}{2} \mathbf{z}_{1}^{T} \mathbf{C}_{0} \mathbf{z}_{1}+\mathbf{z}_{1}{ }^{T} \mathbf{C}_{r} \mathbf{z}_{0}, & H_{\mathbf{z}_{1}}=\mathbf{C}_{0} \mathbf{z}_{1}+\mathbf{C}_{r} \mathbf{z}_{0}, \\
H_{2}\left(\mathbf{z}_{2}\right)=\frac{1}{2} \mathbf{z}_{2}{ }^{T} \mathbf{C}_{0} \mathbf{z}_{2}+\mathbf{z}_{2}{ }^{T} \mathbf{C}_{r} \mathbf{z}_{1}, & H_{\mathbf{z}_{2}}=\mathbf{C}_{0} \mathbf{z}_{2}+\mathbf{C}_{r} \mathbf{z}_{1}, \cdots, \\
H_{m}\left(\mathbf{z}_{m}\right)=\frac{1}{2} \mathbf{z}_{m}{ }^{T} \mathbf{C}_{0} \mathbf{z}_{m}+\mathbf{z}_{m}{ }^{T} \mathbf{C}_{r} \mathbf{z}_{m-1}, & H_{\mathbf{z}_{m}}=\mathbf{C}_{0} \mathbf{z}_{m}+\mathbf{C}_{r} \mathbf{z}_{m-1},
\end{array}
$$

where $\mathbf{C}_{0}=\mathbf{J B}_{0}, \mathbf{C}_{r}=\mathbf{J} \mathbf{B}_{r}$.

Solving the first equation of Eq. (3.4) yields the nominal part of $\mathbf{z}$, namely, $\mathbf{z}_{0}$. Substituting the value of $\mathbf{z}_{0}$ into the second equation of Eq. (3.4) and solving it gives the 
first-order perturbation of $\mathbf{z}$, namely, $\mathbf{z}_{1}$. Then the value of $\mathbf{z}_{i},(i=2,3, \cdots, m)$ can be derived in turn. Thus, $\mathbf{z}$ can be obtained by Eq. (3.2) and the perturbation of $\mathbf{z}$ is expressed as

$$
\mathbf{z}_{r}=\varepsilon \mathbf{z}_{1}+\varepsilon^{2} \mathbf{z}_{2}+\cdots+\varepsilon^{m} \mathbf{z}_{m}+\cdots
$$

The following key lies in how to solve the Hamiltonian canonical equations in Eq. (3.4), which will be introduced in Section 4 .

\subsection{Symplectic structure of the linear Hamiltonian system with perturbations by the perturbation series expansion method}

For the algorithms of Hamiltonian systems, symplectic conservation is a significant criterion to judge whether they are good or not [4]. Therefore, symplectic conservation of the perturbation series expansion method for the linear Hamiltonian system with perturbations is proved mathematically.

As for the symplectic structure of the linear Hamiltonian system with perturbations (3.3), it is also expanded as power series

$$
\omega=\omega_{0}+\varepsilon \omega_{1}+\varepsilon^{2} \omega_{2}+\cdots+\varepsilon^{m} \omega_{m}+\cdots,
$$

where $\omega_{i},(i=0,1, \cdots, m)$ are the symplectic structures of the Hamiltonian canonical equations in Eq. (3.4), respectively.

The symplectic structure of the linear Hamiltonian system with perturbations is also expressed as Eq. (2.6). Substituting Eqs. (3.2) and (3.7) into Eq. (2.6) leads to

$$
\begin{aligned}
& \omega_{0}+\varepsilon \omega_{1}+\varepsilon^{2} \omega_{2}+\cdots+\varepsilon^{m} \omega_{m}+\cdots \\
= & \frac{1}{2} d\left(\mathbf{z}_{0}+\varepsilon \mathbf{z}_{1}+\varepsilon^{2} \mathbf{z}_{2}+\cdots+\varepsilon^{k} \mathbf{z}_{k}+\cdots\right) \wedge \mathbf{J} d\left(\mathbf{z}_{0}+\varepsilon \mathbf{z}_{1}+\varepsilon^{2} \mathbf{z}_{2}+\cdots+\varepsilon^{k} \mathbf{z}_{k}+\cdots\right),
\end{aligned}
$$

where $k$ is a positive integer.

Comparing the coefficients of the same power terms of $\varepsilon$ on each side of Eq. (3.8), we can obtain the symplectic structures of the Hamiltonian canonical equations in Eq. (3.4)

$$
\begin{aligned}
& \varepsilon^{0}: \omega_{0}=\frac{1}{2} d \mathbf{z}_{0} \wedge \mathbf{J} d \mathbf{z}_{0}, \\
& \varepsilon^{1}: \omega_{1}=\frac{1}{2}\left(d \mathbf{z}_{0} \wedge \mathbf{J} d \mathbf{z}_{1}+d \mathbf{z}_{1} \wedge \mathbf{J} d \mathbf{z}_{0}\right), \\
& \varepsilon^{2}: \omega_{2}=\frac{1}{2}\left(d \mathbf{z}_{0} \wedge \mathbf{J} d \mathbf{z}_{2}+d \mathbf{z}_{1} \wedge \mathbf{J} d \mathbf{z}_{1}+d \mathbf{z}_{2} \wedge \mathbf{J} d \mathbf{z}_{0}\right), \cdots, \\
& \varepsilon^{m}: \omega_{m}=\frac{1}{2} \sum_{i=0}^{m} d \mathbf{z}_{i} \wedge \mathbf{J} d \mathbf{z}_{m-i} .
\end{aligned}
$$


The differential forms of the symplectic structures $\omega_{0}, \omega_{1}, \omega_{2}, \cdots, \omega_{m}$ are presented as

$$
\begin{aligned}
& \varepsilon^{0}: \frac{d \omega_{0}}{d t}=\frac{d}{d t}\left(\frac{1}{2} d \mathbf{z}_{0} \wedge \mathbf{J} d \mathbf{z}_{0}\right), \\
& \varepsilon^{1}: \frac{d \omega_{1}}{d t}=\frac{d}{d t}\left[\frac{1}{2}\left(d \mathbf{z}_{0} \wedge \mathbf{J} d \mathbf{z}_{1}+d \mathbf{z}_{1} \wedge \mathbf{J} d \mathbf{z}_{0}\right)\right], \\
& \varepsilon^{2}: \frac{d \omega_{2}}{d t}=\frac{d}{d t}\left[\frac{1}{2}\left(d \mathbf{z}_{0} \wedge \mathbf{J} d \mathbf{z}_{2}+d \mathbf{z}_{1} \wedge \mathbf{J} d \mathbf{z}_{1}+d \mathbf{z}_{2} \wedge \mathbf{J} d \mathbf{z}_{0}\right)\right], \cdots, \\
& \varepsilon^{m}: \frac{d \omega_{m}}{d t}=\frac{d}{d t}\left[\frac{1}{2}\left(\sum_{i=0}^{m} d \mathbf{z}_{i} \wedge \mathbf{J} d \mathbf{z}_{m-i}\right)\right] .
\end{aligned}
$$

From the first equation of Eq. (3.10), similar to that in Section 2.2, we can prove easily that the symplectic structure $\omega_{0}$ holds the symplecticity along the phase flow

$$
\frac{d \omega_{0}}{d t}=0
$$

Let

$$
\mathbf{z}_{t}^{i}=\frac{d \mathbf{z}_{i}}{d t}, \quad i=0,1, \cdots, m
$$

Then, the second equation of Eq. (3.10) can be expressed as

$$
\begin{aligned}
\frac{d \omega_{1}}{d t} & =\frac{1}{2}\left(d \mathbf{z}_{t}^{0} \wedge \mathbf{J} d \mathbf{z}_{1}+d \mathbf{z}_{1} \wedge \mathbf{J} d \mathbf{z}_{t}^{0}+d \mathbf{z}_{0} \wedge \mathbf{J} d \mathbf{z}_{t}^{1}+d \mathbf{z}_{t}^{1} \wedge \mathbf{J} d \mathbf{z}_{0}\right) \\
& =\frac{1}{2}\left(d \mathbf{z}_{1} \wedge \mathbf{J} d \mathbf{z}_{t}^{0}+d \mathbf{z}_{1} \wedge \mathbf{J} d \mathbf{z}_{t}^{0}+d \mathbf{z}_{0} \wedge \mathbf{J} d \mathbf{z}_{t}^{1}+d \mathbf{z}_{0} \wedge \mathbf{J} d \mathbf{z}_{t}^{1}\right) \\
& =d \mathbf{z}_{1} \wedge \mathbf{J} d \mathbf{z}_{t}^{0}+d \mathbf{z}_{0} \wedge \mathbf{J} d \mathbf{z}_{t}^{1} .
\end{aligned}
$$

The variational equations of the first two equations of Eq. (3.4) are

$$
\mathbf{J} d \mathbf{z}_{t}^{0}=H_{\mathbf{z}_{0} \mathbf{z}_{0}}\left(\mathbf{z}_{0}\right) d \mathbf{z}_{0}, \quad \mathbf{J} d \mathbf{z}_{t}^{1}=H_{\mathbf{z}_{1} \mathbf{z}_{1}}\left(\mathbf{z}_{1}\right) d \mathbf{z}_{1} .
$$

Taking the exterior product of the former equation of Eq. (3.14) with $d \mathbf{z}_{1}$ and of the latter equation of Eq. (3.14) with $d \mathbf{z}_{0}$ yield

$$
d \mathbf{z}_{1} \wedge \mathbf{J} d \mathbf{z}_{t}^{0}=d \mathbf{z}_{1} \wedge H_{\mathbf{z}_{0} \mathbf{z}_{0}}\left(\mathbf{z}_{0}\right) d \mathbf{z}_{0}=0, \quad d \mathbf{z}_{0} \wedge \mathbf{J} d \mathbf{z}_{t}^{1}=d \mathbf{z}_{0} \wedge H_{\mathbf{z}_{1} \mathbf{z}_{1}}\left(\mathbf{z}_{1}\right) d \mathbf{z}_{1}=0 .
$$

Thus, we have

$$
\frac{d \omega_{1}}{d t}=d \mathbf{z}_{1} \wedge \mathbf{J} d \mathbf{z}_{t}^{0}+d \mathbf{z}_{0} \wedge \mathbf{J} d \mathbf{z}_{t}^{1}=0
$$

In the similar manner, from the other equations of Eq. (3.10) we can obtain

$$
\frac{d \omega_{2}}{d t}=0, \cdots, \frac{d \omega_{m}}{d t}=0
$$

Eqs. (3.11), (3.16) and (3.17) indicate that the symplectic structures of the Hamiltonian canonical equations in Eq. (3.4) hold the symplecticity, respectively. 


\section{Symplectic difference schemes for Hamiltonian systems}

The symplectic difference schemes for Hamiltonian systems can be adopted to solve the Hamiltonian canonical equations [2].

For the Hamiltonian canonical equations in Eq. (3.4), the Euler centered scheme is presented as

$$
\frac{\mathbf{z}_{i}^{k+1}-\mathbf{z}_{i}^{k}}{\tau}=\mathbf{J}^{-1} H_{\mathbf{z}_{i}}\left(\frac{\mathbf{z}_{i}^{k+1}+\mathbf{z}_{i}^{k}}{2}\right), \quad i=0,1, \cdots, m,
$$

where $\tau$ is the time step.

For the first equation of Eq. (3.4), which is a linear homogeneous Hamiltonian canonical equation, the transition $\mathbf{z}_{0}^{k} \mapsto \mathbf{z}_{0}^{k+1}$ is given by

$$
\mathbf{z}_{0}^{k+1}=\mathbf{F}_{\tau_{0}} \mathbf{z}_{0}^{k}
$$

where

$$
\mathbf{F}_{\tau_{0}}=\left(\mathbf{I}-\frac{\tau}{2} \mathbf{B}_{0}\right)^{-1}\left(\mathbf{I}+\frac{\tau}{2} \mathbf{B}_{0}\right)
$$

Proposition 4.1 ([2]). If $\mathbf{A}$ is infinitesimal symplectic, and $|\mathbf{I}+\mathbf{A}| \neq 0$, then $\mathbf{F}=(\mathbf{I}+\mathbf{A})^{-1}(\mathbf{I}-\mathbf{A})$ is a symplectic matrix, which is called the Cayley transformation of $\mathbf{A}$.

Since $\mathbf{B}_{0}$ is infinitesimal symplectic, $-\frac{\tau}{2} \mathbf{B}_{0}$ is also infinitesimal symplectic, and $\mathbf{F}_{\tau_{0}}$ is the Cayley transformation of the infinitesimal symplectic $-\frac{\tau}{2} \mathbf{B}_{0}$. According to Proposition 4.1, $\mathbf{F}_{\tau_{0}}$ is symplectic and the scheme is symplectic as well. By using the Euler centered scheme for the linear Hamiltonian system to solve the first equation of Eq. (3.4), we can obtain $\mathbf{z}_{0}$.

For the other equations of Eq. (3.4), the mappings $\mathbf{F}_{\tau_{i}}: \mathbf{z}_{i}^{k} \mapsto \mathbf{z}_{i}^{k+1},(i=1, \cdots, m)$ are nonlinear. By differentiation,

$$
\frac{\partial \mathbf{z}_{i}^{k+1}}{\partial \mathbf{z}_{i}^{k}}=\mathbf{I}+\tau \mathbf{J}^{-1} H_{\mathbf{z}_{i} \mathbf{z}_{i}}\left(\frac{\mathbf{z}_{i}^{k+1}+\mathbf{z}_{i}^{k}}{2}\right)\left(\frac{1}{2} \frac{\partial \mathbf{z}_{i}^{k+1}}{\partial \mathbf{z}_{i}^{k}}+\frac{1}{2} \mathbf{I}\right),
$$

where $H_{\mathbf{z}_{i} \mathbf{z}_{i}}\left(\frac{\mathbf{z}_{i}^{k+1}+\mathbf{z}_{i}^{k}}{2}\right)$ is the Hessian matrix of the function $H_{i}\left(\mathbf{z}_{i}\right)$ at point $\mathbf{z}_{i}=\frac{\mathbf{z}_{i}^{k+1}+\mathbf{z}_{i}^{k}}{2}$ and $\frac{\partial \mathbf{z}_{i}^{k+1}}{\partial \mathbf{z}_{i}^{k}}$ is the Jacobian matrix of $\mathbf{F}_{\tau_{i}}$. We have

$$
\mathbf{F}_{\tau_{i}}=\left[\mathbf{I}-\frac{\tau}{2} \mathbf{J}^{-1} H_{\mathbf{z}_{i} \mathbf{z}_{i}}\left(\frac{\mathbf{z}_{i}^{k+1}+\mathbf{z}_{i}^{k}}{2}\right)\right]^{-1}\left[\mathbf{I}+\frac{\tau}{2} \mathbf{J}^{-1} H_{\mathbf{z}_{i} \mathbf{z}_{i}}\left(\frac{\mathbf{z}_{i}^{k+1}+\mathbf{z}_{i}^{k}}{2}\right)\right] .
$$

When $\mathbf{z}_{i}$ remains bounded and by taking $\tau$ to be sufficiently small, we can keep the infinitesimally symplectic matrix $-\frac{\tau}{2} \mathbf{J}^{-1} H_{\mathbf{z z}_{i}}\left(\frac{\mathbf{z}_{i}^{k+1}+\mathbf{z}_{i}^{k}}{2}\right)$ non-exceptional. Then, $\mathbf{F}_{\tau_{i}}$ as a 
Cayley transformation is symplectic. Accordingly, we can solve the other equations of Eq. (3.4) successively by employing the Euler centered scheme for the nonlinear Hamiltonian system to obtain the ith-order perturbation of $\mathbf{z}$, i.e., $\mathbf{z}_{i},(i=1, \cdots, m)$.

To the end, $\mathbf{z}$ and the perturbation of $\mathbf{z}$, namely, $\mathbf{z}_{r}$ can be obtained.

\section{Numerical examples}

In order to demonstrate the validity of the proposed method (abbreviated as PSEM), three numerical examples are provided in this section, including a second-order linear Hamiltonian system, a Bernoulli-Euler cantilever beam and a fully clamped composite laminate. For the sake of comparison, the results obtained by the proposed method are compared with those obtained by the exact method, the second-order and the fourthorder Runge-Kutta algorithms (EM, RK2 and RK4, respectively).

\subsection{Second-order linear Hamiltonian system}

Firstly, consider a mathematical example, a second-order linear Hamiltonian system expressed as

$$
\frac{d}{d t}\left(\begin{array}{l}
p \\
q
\end{array}\right)=\mathbf{B}\left(\begin{array}{l}
p \\
q
\end{array}\right)
$$

with the initial condition $(p(0), q(0))^{T}=(1,1)^{T}$, where $\mathbf{B}$ is infinitesimal symplectic.

The nominal Hamiltonian function $H_{0}$ and the corresponding infinitesimal symplectic matrix $\mathbf{B}_{0}$ are presented as

$$
H_{0}=-\frac{1}{2} p^{2}-\frac{5}{2} q^{2}-p q, \quad \mathbf{B}_{0}=\left(\begin{array}{cc}
1 & 5 \\
-1 & -1
\end{array}\right)
$$

The analytic solution to the nominal system is represented as

$$
\left(\begin{array}{l}
p(t) \\
q(t)
\end{array}\right)=\left(\begin{array}{c}
\cos (2 t)+3 \sin (2 t) \\
-\sin (2 t)+\cos (2 t)
\end{array}\right)
$$

Suppose there is a small perturbation in the infinitesimal symplectic matrix $\mathbf{B}$ given as

$$
\mathbf{B}_{r}=\left(\begin{array}{cc}
0 & 0.2 \\
0.1 & 0
\end{array}\right)
$$

Hence the Hamiltonian function $H$ in the perturbed system can be expressed as

$$
H=-\frac{0.9}{2} p^{2}-\frac{5.2}{2} q^{2}-p q
$$




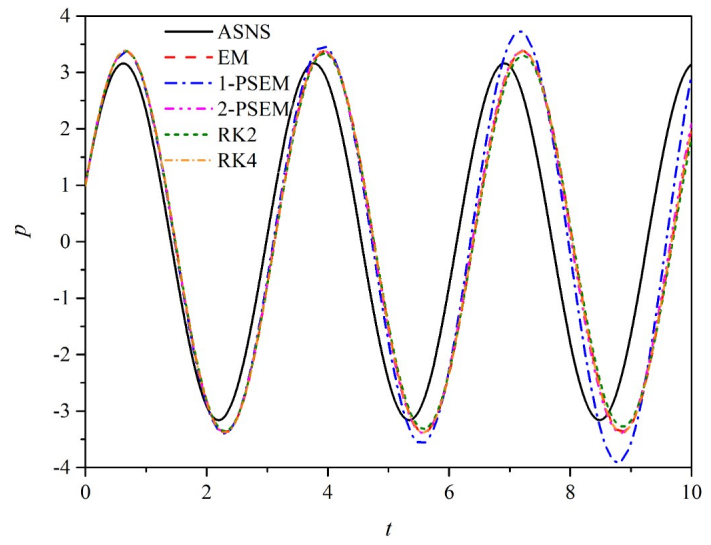

(a) $p$

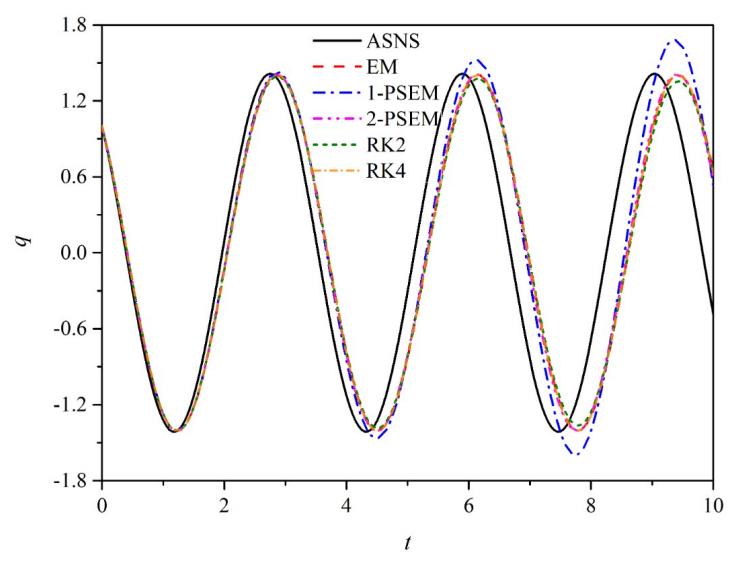

(b) $q$

Figure 1: ASNS $p_{0}, q_{0}$ and the perturbed system $p, q$ obtained by EM, PSEM and RK of the second-order linear Hamiltonian system.

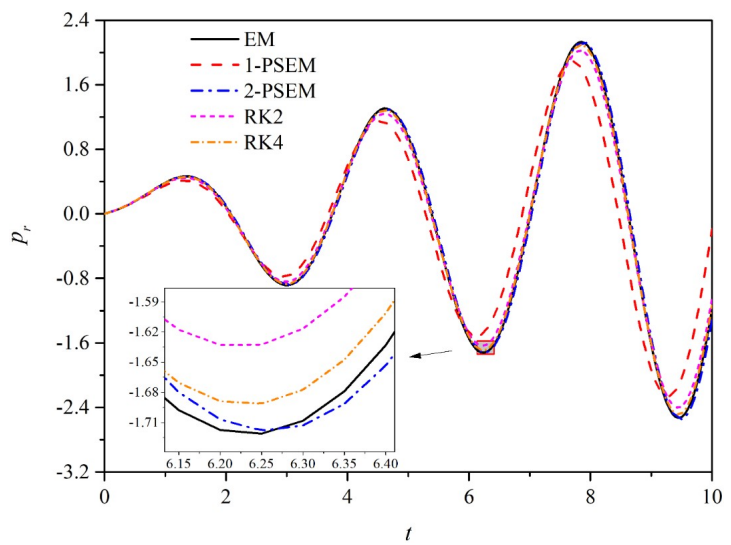

(a) $p_{r}$

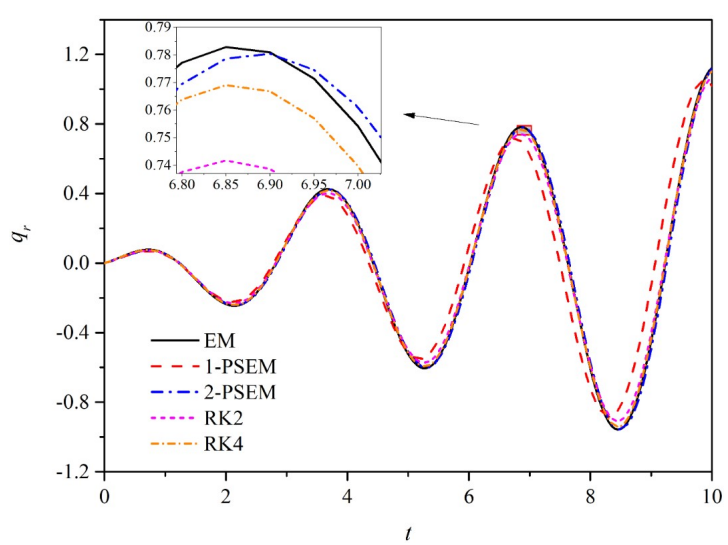

(b) $q_{r}$

Figure 2: Perturbations $p_{r}, q_{r}$ obtained by EM, PSEM and RK of the second-order linear Hamiltonian system.

Here the first two order methods of PSEM (1-PSEM and 2-PSEM, respectively) are taken into consideration and the time step is set to be $\Delta t=0.05$. The analytic solutions to the nominal system (ASNS) $p_{0}, q_{0}$ and the numerical results of the perturbed system $p, q$ obtained by EM, PSEM and RK are exhibited in Fig. 1. In order to investigate the effects of the small perturbation on the response clearly, the perturbations $p_{r}, q_{r}$, which represent the deviations of the perturbed system $p, q$ from the nominal system $p_{0}, q_{0}$, are plotted in Fig. 2. The numerical results at $t=4$ and $t=8$ are listed in Table 1 .

The figures and table show that although the perturbation in $\mathbf{B}$ is very small, both amplitude and phase of the system increase compared to the nominal system. The deviations between the nominal system and the perturbed system indicate that the perturba- 
Table 1: ASNS $p_{0}, q_{0}$, the perturbed system $p, q$ and the perturbations $p_{r}, q_{r}$ obtained by EM, PSEM and RK at $t=4$ and $t=8$ of the second-order linear Hamiltonian system.

\begin{tabular}{||cccccccc||}
\hline \multicolumn{1}{||c}{$t=4$} & $t=8$ & & & $t=4$ & $t=8$ \\
\hline$p_{0}$ & ASNS & 2.8320 & -1.7868 & $q_{0}$ & ASNS & -1.1292 & -0.6863 \\
\hline & EM & 3.3614 & 0.2467 & & EM & -0.7878 & -1.2922 \\
& 1-PSEM & 3.4279 & -0.2321 & & 1-PSEM & -0.8507 & -1.4077 \\
& 2-PSEM & 3.3569 & 0.2791 & $q$ & 2-PSEM & -0.7794 & -1.2839 \\
& RK2 & 3.3258 & 0.1280 & & RK2 & -0.8089 & -1.2561 \\
& RK4 & 3.3590 & 0.2071 & & RK4 & -0.7947 & -1.2856 \\
\hline & EM & 0.5294 & 2.0335 & & EM & 0.3414 & -0.6059 \\
& 1-PSEM & 0.5959 & 1.5547 & & 1-PSEM & 0.2785 & -0.7214 \\
$p_{r}$ & 2-PSEM & 0.5249 & 2.0659 & \multirow{2}{*}{$q_{r}$} & 2-PSEM & 0.3498 & -0.5976 \\
& RK2 & 0.4938 & 1.9148 & & RK2 & 0.3203 & -0.5698 \\
& RK4 & 0.5270 & 1.9939 & & RK4 & 0.3345 & -0.5993 \\
\hline
\end{tabular}

tion has a distinct effect on the response. Hence, we cannot ignore the existence of the perturbation. As the order of PSEM increases, the numerical results obtained by PSEM are gradually closer to those obtained by EM, indicating that the accuracy of PSEM is continuously improved. Besides, 2-PSEM can give better accuracy than RK4 with high precision.

Moreover, the Hamiltonian function $H$ in the perturbed system obtained by PSEM, RK2 and RK4 are exhibited in Fig. 3. We can see obviously that the Hamiltonian function remains $H=-4.15$ unchanged during the process of numerical calculation via PSEM. Nevertheless, although RK4 has high precision, its Hamiltonian function $H$ keeps decreasing. Meanwhile, the Hamiltonian function $H$ of RK2 decreases at a faster rate.

Fig. 4 plots the phase diagrams of $(p, q)$ of the perturbed system obtained by PSEM, RK2 and RK4. The phase diagram of $(p, q)$ obtained by PSEM keeps the shape and area

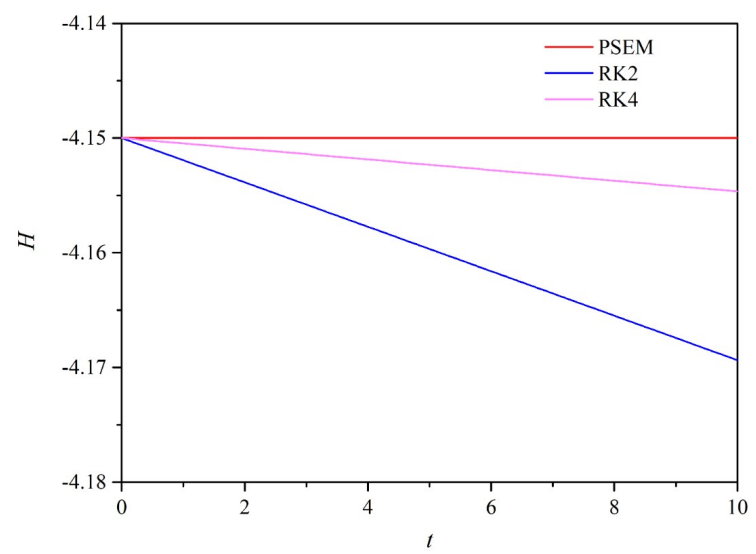

Figure 3: Hamiltonian function $H$ obtained by PSEM, RK2 and RK4 of the second-order linear Hamiltonian system. 


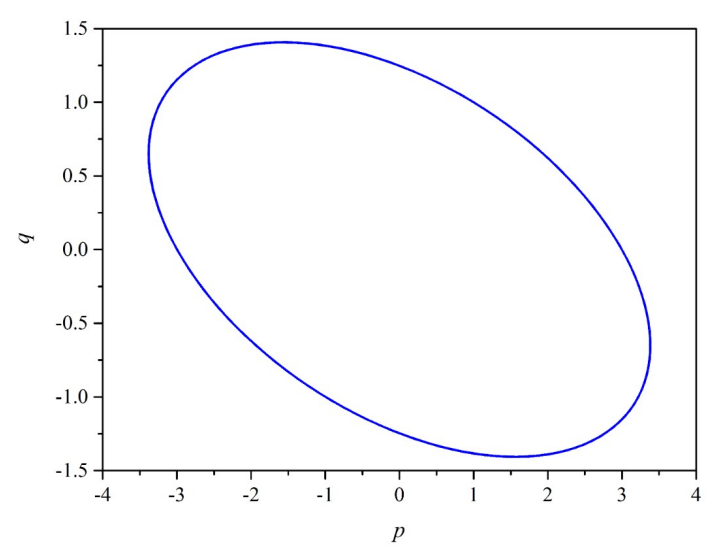

(a) PSEM

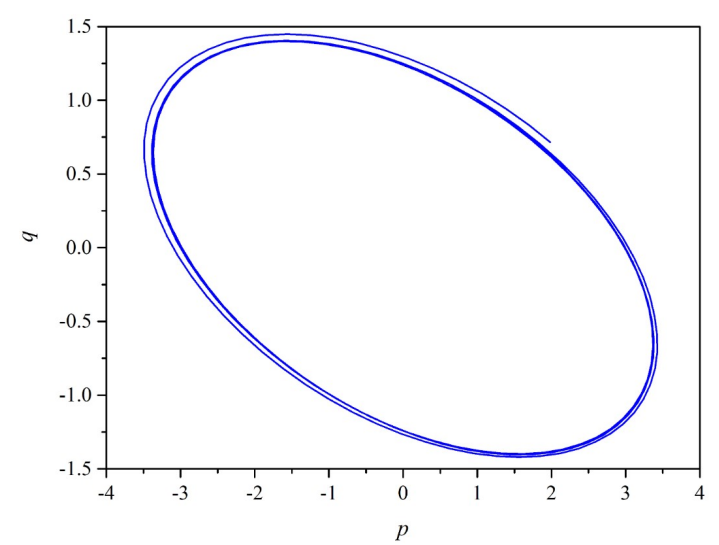

(b) RK2

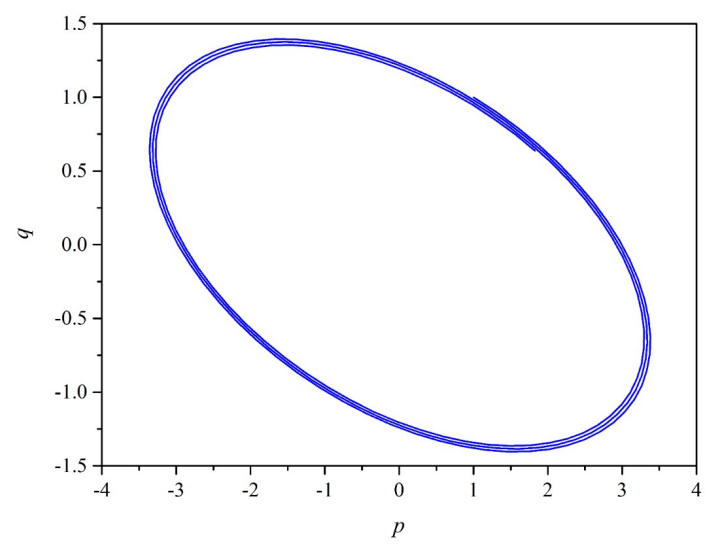

(c) RK4

Figure 4: Phase diagrams of $(p, q)$ obtained by PSEM, RK2 and RK4 of the second-order linear Hamiltonian system.

of the ellipse unchanged while there exist apparent drifts in the phase diagrams of $(p, q)$ obtained by RK2 and RK4 respectively, which are caused by their dissipative nature. The above phenomena illustrate the symplectic conservation of PSEM.

Thus, the mathematical example verifies the feasibility, accuracy and symplectic conservation of PSEM.

\subsection{Bernoulli-Euler cantilever beam}

Next, we demonstrate the applicability of PSEM in a structural dynamic system. Consider a Bernoulli-Euler cantilever beam with 11 nodes and 10 elements as shown in Fig. 5 to certify the effectiveness of the proposed method applied in structural dynamic response estimation. The beam is characterized by the following geometrical properties: 


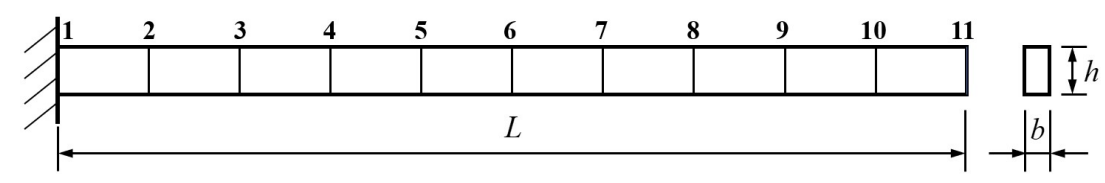

Figure 5: Bernoulli-Euler cantilever beam.

the length $L=5 \mathrm{~m}$, the rectangular cross-sectional area with the width $b=0.1 \mathrm{~m}$ and the height $h=0.2 \mathrm{~m}$.

Due to the inevitable dispersions and measurement errors in materials, there exist perturbations in the material properties. The nominal and perturbed parts of the Young's modulus and the mass density are, respectively

$$
E_{0}=210 \mathrm{GPa}, \quad E_{r}=5 \% \times E_{0}, \quad \rho_{0}=7800 \mathrm{~kg} / \mathrm{m}^{3}, \quad \rho_{r}=5 \% \times \rho_{0} .
$$

The response caused by the force $F=200 \mathrm{~N}$ acting on the vertical direction of the node 11 is taken as the initial condition of the free vibration of the cantilever beam.

The differential equation of motion of the system is expressed as

$$
\mathbf{M} \ddot{\mathbf{x}}+\mathbf{K x}=0,
$$

where $\mathbf{M}$ denotes the global mass matrix, which is related to the mass density, and $\mathbf{K}$ is the global stiffness matrix related to the Young's modulus.

Let $\mathbf{y}=\mathbf{M} \dot{\mathbf{x}}$, then the differential equation of motion can be written in the matrix form as

$$
\left(\begin{array}{c}
\dot{\mathbf{x}} \\
\dot{\mathbf{y}}
\end{array}\right)=\left(\begin{array}{cc}
\mathbf{0} & \mathbf{M}^{-1} \\
-\mathbf{K} & \mathbf{0}
\end{array}\right)\left(\begin{array}{l}
\mathbf{x} \\
\mathbf{y}
\end{array}\right)
$$

Since

$$
\mathbf{B}=\left(\begin{array}{cc}
\mathbf{0} & \mathbf{M}^{-1} \\
-\mathbf{K} & \mathbf{0}
\end{array}\right)
$$

satisfies

$$
\mathbf{J B}+\mathbf{B}^{T} \mathbf{J}=0,
$$

B is infinitesimal symplectic. Eq. (5.8) is the Hamiltonian canonical equation of the system.

PSEM and RK are applied for evaluating the deflection responses of the node 6 and 11 in the vertical direction of the perturbed cantilever beam. The time step is set to be $\Delta t=0.002 \mathrm{~s}$, and the numerical results of the deflection responses of the node 6 and 11 obtained by EM and PSEM are plotted in Figs. 6(a)-(b), respectively. The results of the node 6 and 11 at $t=1.6 \mathrm{~s}$ and $t=2.8 \mathrm{~s}$ are tabulated in Table 2 .

In terms of the accuracy of PSEM, Fig. 6 and Table 2 display that the results obtained by PSEM are in better agreement with those obtained by EM with the increase of the 


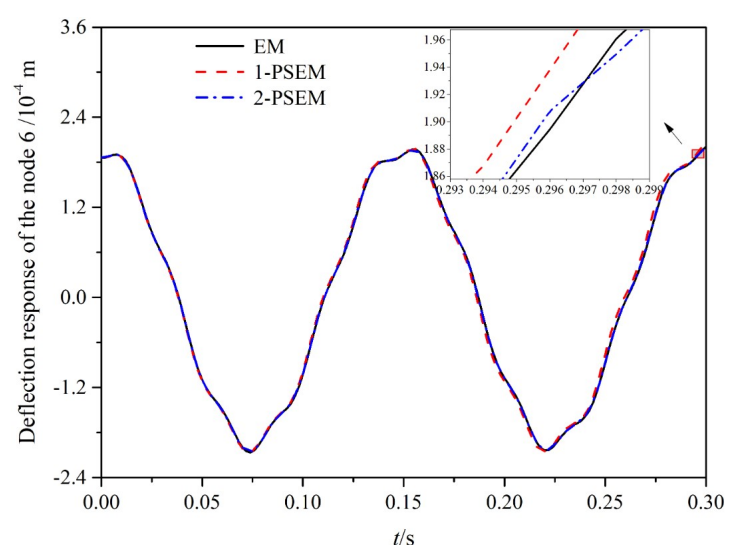

(a) node 6

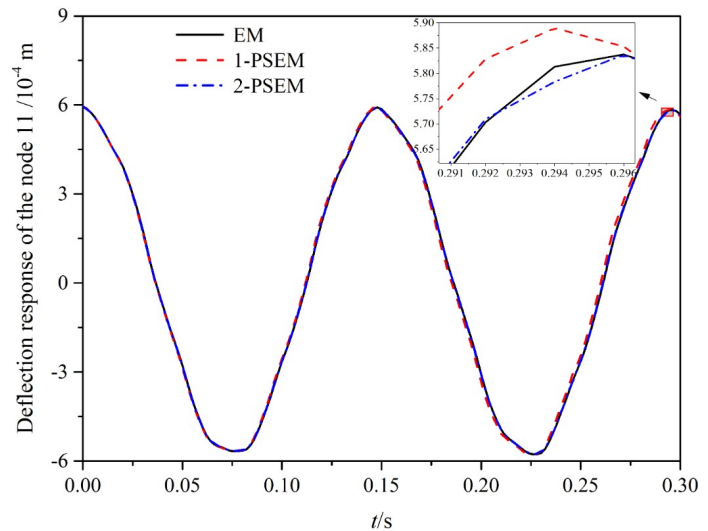

(b) node 11

Figure 6: Deflection responses of the node 6 and 11 obtained by EM and PSEM of the perturbed Bernoulli-Euler cantilever beam.

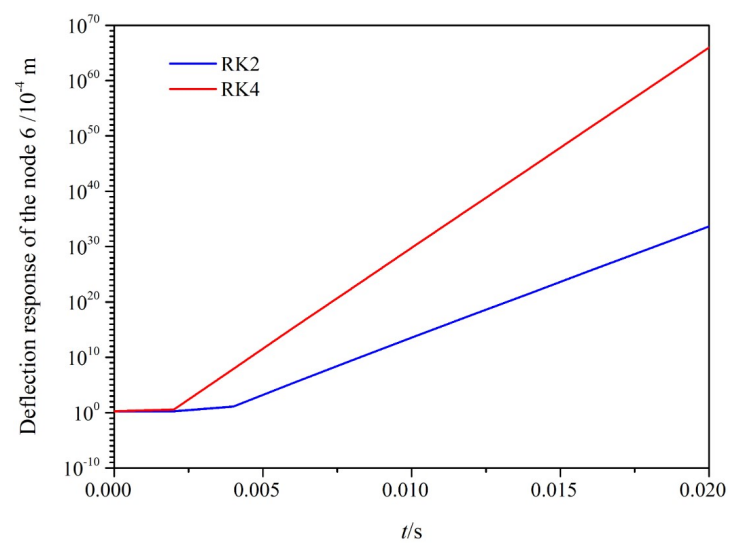

(a) node 6

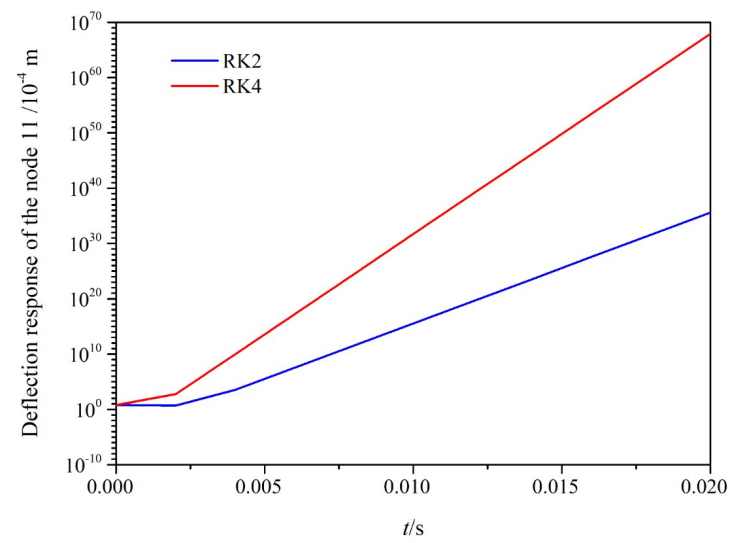

(b) node 11

Figure 7: Deflection responses of the node 6 and 11 obtained by RK of the perturbed Bernoulli-Euler cantilever beam.

order. That is to say, the results calculated by PSEM are becoming more accurate as the order increases and 2-PSEM is of very high accuracy for the requirements.

However, it is worth noting that the numerical results calculated by RK2 and RK 4 are exponentially divergent under the condition of the set time step in a short time interval $t \in[0,0.020] \mathrm{s}$ as shown in Fig. 7. Only when the time step is extremely small like $\Delta t=10^{-6} \mathrm{~s}$, satisfactory results can be achieved. As is known, RK4 is common used due to its high accuracy and stability, but is not applicable for this example. On the contrary, PSEM shows an outstanding advantage in terms of stability. This phenomenon highlights the superiority of PSEM. 


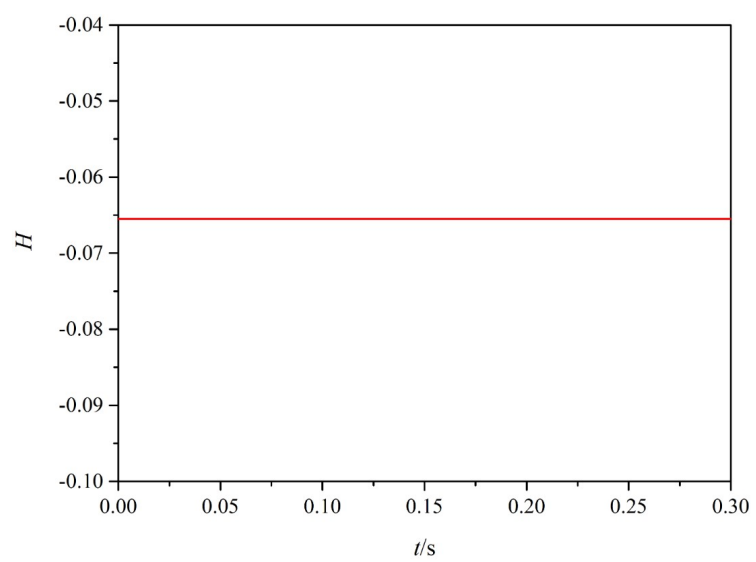

Figure 8: Hamiltonian function $H$ obtained by PSEM of the perturbed Bernoulli-Euler cantilever beam.

Table 2: Deflection responses of the node 6 and 11 obtained by EM and PSEM at $t=1.6 \mathrm{~s}$ and $t=2.8 \mathrm{~s}$ of the perturbed Bernoulli-Euler cantilever beam $\left(10^{-4} \mathrm{~m}\right)$.

\begin{tabular}{||c|c|cc||}
\hline & & $t=1.6 \mathrm{~s}$ & $t=2.8 \mathrm{~s}$ \\
\hline \multirow{3}{*}{ Node 6 } & EM & 1.8543 & 1.5059 \\
& 1-PSEM & 1.8215 & 1.5947 \\
& 2-PSEM & 1.8512 & 1.5046 \\
\hline \multirow{3}{*}{ Node 11 } & EM & 5.0644 & 3.9633 \\
& 1-PSEM & 4.9855 & 4.1786 \\
& 2-PSEM & 5.0603 & 3.9655 \\
\hline
\end{tabular}

Furthermore, the Hamiltonian function $H$ obtained by PSEM is exhibited in Fig. 8. As can be seen, the Hamiltonian function $H$ remains constant, which indicates the symplectic conservation of PSEM.

\subsection{Composite laminate with fully clamped}

The last example concerns a fully clamped composite laminate with the side length $L=$ $100 \mathrm{~mm}$ and the mass density $\rho=1500 \mathrm{~kg} / \mathrm{m}^{3}$ as shown in Fig. 9, which is made of five layers of orthotropic materials with the laying angle of $\left(0^{\circ}, 90^{\circ}, 0^{\circ}, 90^{\circ}, 0^{\circ}\right)$ and the thickness of each layer of $t=0.4 \mathrm{~mm}$.

The dispersions in the material properties introduced by the material production and measurement process are unavoidable. The nominal and perturbed parts of the material properties are

$$
\begin{array}{llll}
E_{1}=38.6 \mathrm{GPa}, & E_{1 r}=5 \% \times E_{1}, & E_{2}=8.27 \mathrm{GPa}, & E_{2 r}=5 \% \times E_{2}, \\
v_{21}=0.26, & v_{21 r}=10 \% \times v_{21}, & G_{12}=4.14 \mathrm{GPa}, & G_{12 r}=7 \% \times G_{12} .
\end{array}
$$

The response caused by the force $F=-50 \mathrm{~N}$ acting on the centre of the laminate in $z$ - 


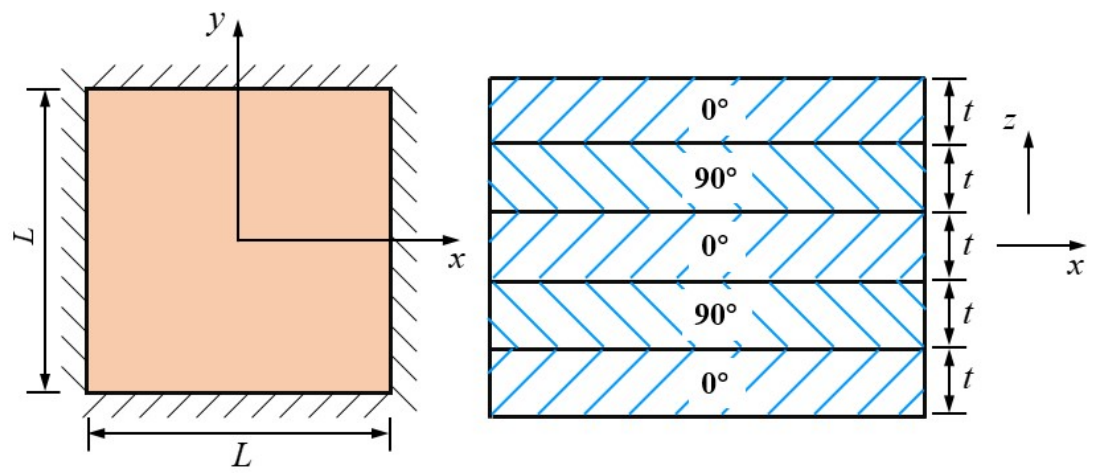

Figure 9: Composite laminate with fully clamped.

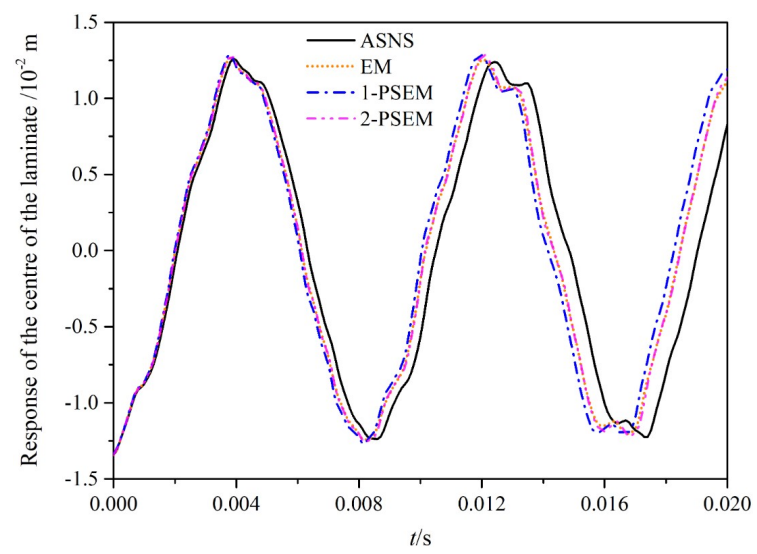

Figure 10: Response of the centre of the laminate obtained by EM and PSEM of the composite laminate.

direction is taken as the initial condition of the free vibration of the laminate. The 4node rectangular thin plate element is utilized and the laminate is divided into 16 equal elements.

The Hamiltonian canonical equation of the laminate is similar to that of the cantilever beam. The response of the centre of the laminate in z-direction is considered. The first two order methods of PSEM are also adopted and the time step is $\Delta t=1 \times 10^{-4} \mathrm{~s}$. The numerical results of the response of the centre of the laminate in the perturbed system obtained by EM and PSEM are plotted in Fig. 10. ASNS are also exhibited in Fig. 10. The numerical results of the response and perturbations at $t=0.010 \mathrm{~s}$ and $t=0.018 \mathrm{~s}$ are listed in Table 3. The Hamiltonian function $H$ in the perturbed system obtained by PSEM is shown in Fig. 11.

From the figures and table, it can be seen obviously that due to the presence of the perturbed parts in the material properties, there are deviations between the perturbed system and the nominal system, even quite different in some time. Thus, we must con- 


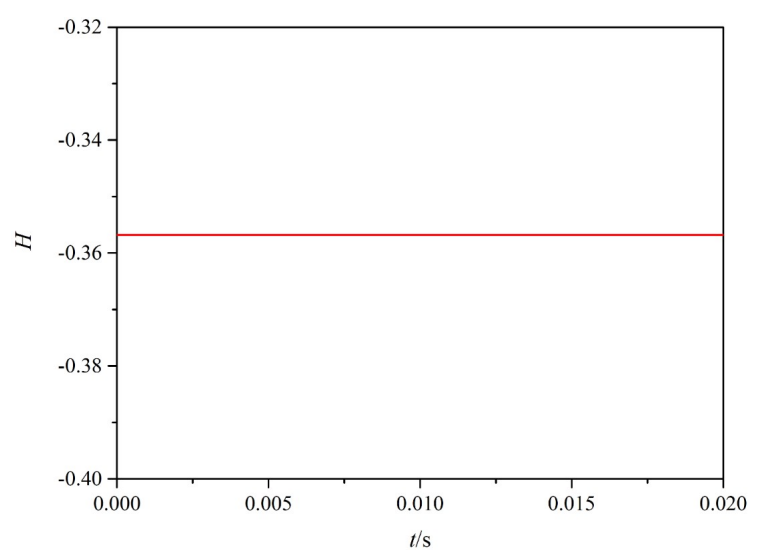

Figure 11: Hamiltonian function $H$ obtained by PSEM of the composite laminate.

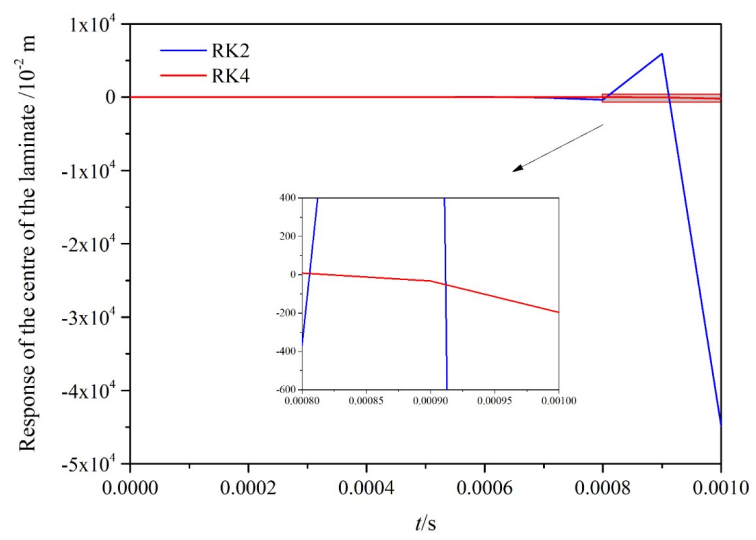

Figure 12: Response of the centre of the laminate obtained by RK of the composite laminate.

sider the effects of perturbations. In addition, as for the numerical results of the perturbed system, the results obtained by 2-PSEM are in excellent accordance with those obtained by EM. What's more, the Hamiltonian function $H$ obtained by PSEM remains unchanged. Therefore, it is illustrated again that PSEM is of high accuracy and symplectic conservation.

By virtue of RK, the numerical results calculated by RK2 and RK4 are divergent in a very short time interval $t \in[0,0.001] \mathrm{s}$ as plotted in Fig. 12. Only under the condition that the time step is extremely small, namely, $\Delta t=1 \times 10^{-5} \mathrm{~s}$ for RK2 and $\Delta t=4 \times 10^{-5} \mathrm{~s}$ for RK4, we can obtain the reasonable results in $t \in[0,0.020] \mathrm{s}$. Since the calculation time of each step of PSEM and RK may be different, the comparison of the calculation time of PSEM and RK at different time steps is given in Table 4, where the maximum error denotes the maximum relative error compared to the results via EM. The computation is performed in MATLAB R2014a on a 2.50GHz Intel Core i5-7200U CPU computer. Compared with 
Table 3: ASNS, the perturbed value and the perturbations of the response of the centre of the laminate obtained by EM and PSEM at $t=0.010 \mathrm{~s}$ and $t=0.018 \mathrm{~s}$ of the composite laminate $\left(10^{-2} \mathrm{~m}\right)$.

\begin{tabular}{||cccc||}
\hline & & $t=0.010 \mathrm{~s}$ & $t=0.018 \mathrm{~s}$ \\
\hline \multirow{4}{*}{ Response } & ASNS & -0.5652 & -0.8049 \\
& EM & -0.1908 & -0.4215 \\
& 1-PSEM & -0.0499 & -0.2400 \\
& 2-PSEM & -0.2008 & -0.4117 \\
\hline \multirow{3}{*}{ Perturbations } & EM & 0.3744 & 0.3834 \\
& 1-PSEM & 0.5153 & 0.5649 \\
& 2-PSEM & 0.3644 & 0.3932 \\
\hline
\end{tabular}

Table 4: Comparison of the calculation time of PSEM and RK at different time steps of the composite laminate.

\begin{tabular}{||cccc||}
\hline Method & Time step (s) & Calculation time (s) & Maximum error $\left(10^{-4} \mathrm{~m}\right)$ \\
2-PSEM & $1 \times 10^{-4}$ & 8.23 & 0.62 \\
RK2 & $1 \times 10^{-5}$ & 10.81 & 1.04 \\
RK4 & $4 \times 10^{-5}$ & 9.48 & 2.25 \\
\hline
\end{tabular}

RK, PSEM can provide the results with a higher precision under a larger time step, which can shorten the calculation time. In summary, PSEM enjoys a clear advantage in high precision, high efficiency and strong stability.

\section{Conclusions}

This paper proposes a novel and effective symplectic conservative numerical method for linear Hamiltonian systems with perturbations. Due to the existence of perturbations, even small ones may have a significant impact on the response. Under the circumstances, the effects of perturbations of linear Hamiltonian systems are taken into consideration and a perturbation series expansion method (PSEM) is presented to predict the dynamic response. By expanding the solution of the linear Hamiltonian system with perturbations in an asymptotic series with a small parameter, a series of Hamiltonian canonical equations are derived and then the symplectic conservation is demonstrated based on the property of the exterior product. The solution can be obtained ultimately by adopting the symplectic difference schemes to solve a series of the Hamiltonian canonical equations.

Three numerical examples are carried out to investigate the validity, symplectic conservation and engineering applicability of PSEM. Compared with RK, the highly precise results can be obtained by PSEM at a larger time step, especially under the case that RK fails. However, satisfactory results can be obtained by RK only when the time step is extremely small. Thus, PSEM can improve the computing efficiency greatly. In addition, the accuracy of PSEM increases with the increase of the order and 2-PSEM can give a satisfactory accuracy. Furthermore, both the mathematical and engineering examples indicate that the numerical results obtained by PSEM can keep the Hamiltonian function as 
well as the shape and area of the phase diagram unchanged, which indicates that PSEM is symplectic conservative.

Overall, PSEM shows the superior performance in accuracy, efficiency and stability, especially symplectic conservation for solving linear Hamiltonian systems with perturbations. Therefore, PSEM can play a significant role in dynamic response estimation of linear Hamiltonian systems. PSEM serves as a modified numerical model which aims to realize a more reliable and precise prediction of dynamic response evolution by properly accounting for perturbations. Thus, PSEM can become a powerful tool to analyze the effects of a small perturbation of parameters on dynamic response of structures.

\section{Acknowledgements}

The authors would like to thank the National Nature Science Foundation of China (No. 11772026), the Defense Industrial Technology Development Program (Nos. JCKY2016204B101, JCKY2018601B001), the Beijing Municipal Science and Technology Commission via project (No. Z191100004619006), and the Beijing Advanced Discipline Center for Unmanned Aircraft System for the financial supports.

\section{References}

[1] K. Feng AND M. Z. QIN, The symplectic methods for the computation of Hamiltonian equations, Numerical Methods for Partial Differential Equations, Springer Berlin Heidelberg, 1987, pp. $1-37$.

[2] K. Feng And M. Z. QIN, Symplectic Geometric Algorithms for Hamiltonian Systems, Zhejiang Publishing United Group, Zhejiang Science and Technology Publishing House, Hangzhou and Springer-Verlag Berlin Heidelberg, 2010.

[3] J. X. CAI, Y. S. WANG AND H. LIANG, Local energy-preserving and momentum-preserving algorithms for coupled nonlinear Schrödinger system, J. Comput. Phys., 239 (2013), pp. 30-50.

[4] P. WANG AND C. HUANG, Structure-preserving numerical methods for the fractional Schrödinger equation, Appl. Numer. Math., 129 (2018), pp. 137-158.

[5] C. C. Chen, J. L. Hong AND C. SIM ET AL., Energy and quadratic invariants preserving (EQUIP) multi-symplectic methods for Hamiltonian wave equations, J. Comput. Phys., 418 (2020), 109599.

[6] Z. J. SHANG, KAM theorem of symplectic algorithms for Hamiltonian systems, Numerische Mathematik, 83(3) (1999), pp. 477-496.

[7] E. Hairer, S. E. Norsett AND G. WANner, Solving Ordinary Differential Equations I: Nonstiff Problems, Springer-Verlag, Berlin, 1993.

[8] E. Hairer, C. Lubich AND G. WANNER, Geometric Numerical Integration: StructurePreserving Algorithms for Ordinary Differential Equations, Springer-Verlag, Berlin, 2006.

[9] A. CRISTINA, Explicit pseudo-symplectic methods based on generating functions for stochastic Hamiltonian systems, J. Comput. Appl. Math., 373 (2020), 112433.

[10] X. Y. NIU, J. B. CUI, AND J. L. HONG, Explicit pseudo-symplectic methods for stochastic Hamiltonian systems, BIT Numer. Math., 58(1) (2018), pp. 163-178. 
[11] K. FENG, On difference schemes and symplectic geometry, Proceedings of the 1984 Beijing Symposium on Differential Geometry and Differential Equations, Beijing Science Press, 1984, pp. 42-58.

[12] A. KHAng, D. P. Howsmon And E. Lejeune ET AL., Multi-scale modeling of the heart valve interstitial cell, Multi-scale Extracellular Matrix Mechanics and Mechanobiology, Springer Nature Switzerland AG, 2020, pp. 21-53.

[13] J. M. SANZ-SERNA, Runge-Kutta schemes for Hamiltonian systems, BIT Numer. Math., 28(4) (1988), pp. 877-883.

[14] F. M. LASAgni, Canonical Runge-Kutta methods, Z. Angew. Math. Phys., 39(6) (1988), pp. 952-953.

[15] Y. B. SURIS, On the conservation of the symplectic structure in the numerical solution of Hamiltonian systems, Numerical Solution of Ordinary Differential Equations, Keldysh Institute of Applied Mathematics, USSR Academy of Sciences, Moscow, 1988, pp. 148-160.

[16] G. Sun, Symplectic partitioned Runge-Kutta methods, J. Comput. Math., 11(4) (1993), pp. 365372.

[17] T. J. BRIDGES, Multisymplectic structures and wave propagation, Mathematical Proceedings of Cambridge Philosophical Society, 121(1) (1997), pp. 147-190.

[18] S. REICH, Multi-symplectic Runge-Kutta collocation methods for Hamiltonian wave equations, J. Comput. Phys., 157(2) (2000), pp. 473-499.

[19] Z. J. SUN AND Z. M. WU, Meshless conservative scheme for multivariate nonlinear Hamiltonian PDEs, J. Sci. Comput., 76(2) (2018), pp. 1168-1187.

[20] Z. J. SUN, A meshless symplectic method for two-dimensional nonlinear Schrödinger equations based on radial basis function approximation, Eng. Anal. Bound. Elem., 104 (2019), pp. 1-7.

[21] W. S. TANG, A note on continuous-stage Runge-Kutta methods, Appl. Math. Comput., 339 (2018), pp. 231-241.

[22] W. S. TANG, Y. J. SUN AND J. J. ZHANG, High order symplectic integrators based on continuousstage Runge-Kutta-Nystrom methods, Appl. Math. Comput., 361 (2019), pp. 670-679.

[23] J. J. WANG, Symplectic-preserving Fourier spectral scheme for space fractional Klein-GordoSchrödinger equations, Numer. Methods Partial Differential Eq., 37(2) (2021), pp. 1030-1056.

[24] M. F. FEI, C. M. HuANG AND P. D. WANG, Error estimates of structure-preserving Fourier pseudospectral methods for the fractional Schrödinger equation, Numer. Methods Partial Differential Eq., 36(2) (2020), pp. 369-393.

[25] X. J. ZHANG, W. J. HUANG AND E. LUO, A symplectic algorithm for dynamic response analysis of the Timoshenko beam, Acta Sci. Natur. Univ. Sunyatseni, 4 (2010), pp. 33-37.

[26] W. P. Hu, Z. C. DENG AND H. J. OuYANG, Generalized multi-symplectic method for dynamic responses of continuous beam under moving load, Int. J. Appl. Mech., 5(3) (2013), 1350033.

[27] W. H. Li AND H. Y. SUN, A symplectic method for dynamic response of structures, Appl. Mech. Materials, 724 (2015), pp. 7-11.

[28] D. D. YANG, J. F. HuANG AND W. J. ZHAO, A quasi-dynamic model and a symplectic algorithm of super slender Kirchhoff rod, Int. J. Model. Simul. Sci. Comput., 8(3) (2017), 1750037.

[29] Y. F. XING AND H. M. ZHANG AND Z. K. WANG, Highly precise time integration method for linear structural dynamic analysis, Int. J. Numer. Methods Eng., 116(8) (2018), pp. 505-529.

[30] R. D. ZHANG, L. P. YANG AND W. W. CAI, Symplectic Runge-Kutta method based numerical solution for the Hamiltonian model of spacecraft relative motion, Lecture Notes Electrical Eng., 459 (2019), pp. 2142-2153.

[31] H. J. PENG, N. N. SONG AND Z. Y. KAN, A novel nonsmooth dynamics method for multibody systems with friction and impact based on the symplectic discrete format, Int. J. Numer. Methods 
Eng., 121(7) (2020), pp. 1530-1557.

[32] H. J. PENG, N. N. SONG AND Z. Y. KAN, A nonsmooth contact dynamic algorithm based on the symplectic method for multibody system analysis with unilateral constraints, Multibody Syst. Dyn., 49(2) (2020), pp. 119-153.

[33] L. WANG, Y. J. MA AND Y. W. YANG ET AL., Structural design optimization based on hybrid time-variant reliability measure under non-probabilistic convex uncertainties, Appl. Math. Model., 69 (2019), pp. 330-354.

[34] C. XiOng, L. Wang AND G. H. LiU et Al., An iterative dimension-by-dimension method for structural interval response prediction with multidimensional uncertain variables, Aerosp. Sci. Technol., 86 (2019), pp. 572-581.

[35] C. WANG AND H. G. MATTHIES, Hybrid evidence-and-fuzzy uncertainty propagation under a dual-level analysis framework, Fuzzy Sets Syst., 367 (2019), pp. 51-67.

[36] C. WANG AND H. G. MATTHIES, Random model with fuzzy distribution parameters for hybrid uncertainty propagation in engineering systems, Comput. Methods Appl. Mech. Eng., 359 (2020), 112673.

[37] C. WANG AND H. G. MATTHIES, A comparative study of two interval-random models for hybrid uncertainty propagation analysis, Mech. Syst. Signal Process., 136 (2020), 106531.

[38] Z. P. QIU AND P. B. WANG, Parameter vertex method and its parallel solution for evaluating the dynamic response bounds of structures with interval parameters, Sci. China Phys. Mech. Astron., 61(6) (2018), pp. 66-78.

[39] Y. S. LIU, X. J. WANG AND L. WANG, A dynamic evolution scheme for structures with interval uncertainties by using bidirectional sequential Kriging method, Comput. Methods Appl. Mech. Eng., 348 (2019), pp. 712-729.

[40] C. WANG AND H. G. MATthiEs, A modified parallelepiped model for non-probabilistic uncertainty quantification and propagation analysis, Comput. Methods Appl. Mech. Eng., 369 (2020), 113209.

[41] Z. P. QIU AND N. JIANG, An ellipsoidal Newton's iteration method of nonlinear structural systems with uncertain-but-bounded parameters, Comput. Methods Appl. Mech. Eng., 373 (2021), 113501.

[42] A. H. NAYFEH, Introduction to Perturbation Techniques, A Wiley-Interscience Publication, John Wiley and Sons, New York, 1993.

[43] M. H. HolmeS, Introduction to Perturbation Methods, Springer Science and Business Media, New York, 1995.

[44] Z. P. QIU AND Z. S. ZHANG, Crack propagation in structures with uncertain-but-bounded parameters via interval perturbation method, Theor. Appl. Fracture Mech., 98 (2018), pp. 95-103.

[45] H. C. QIU, A modified stochastic perturbation algorithm for closely-spaced eigenvalues problems based on surrogate model, Struct. Multidiscip. O., 56(2) (2017), pp. 249-270.

[46] X. Z. LI, W. M. XIANG AND H. C. FU ET AL., Iterative perturbation methods for isolation structure dynamic model responses calculation with non-classical damping and its correlation analysis, Adv. Mater. Res., 415-417 (2012), pp. 2172-2179.

[47] G. Sliva, A. Brezillon And J. M. CAdOU ET Al., A study of the eigenvalue sensitivity by homotopy and perturbation methods, J. Comput. Appl. Math., 234(7) (2010), pp. 2297-2302.

[48] Z. P. QIU AND Y. N. ZHENG, Predicting fatigue crack growth evolution via perturbation series expansion method based on the generalized multinomial theorem, Theor. Appl. Fracture Mech., 86 (2016), pp. 361-369.

[49] Z. P. QIU AND J. J. ZHU, The perturbation series method based on the logarithm equation for fatigue crack growth prediction, Theor. Appl. Fracture Mech., 103 (2019), 102239. 
[50] Z. P. QIU AND N. JIANG, Comparative study of stochastic and interval non-homogeneous linear Hamiltonian systems and their applications, J. Theor. Appl. Mech., 52(1) (2020), pp. 60-72 (in Chinese).

[51] Z. P. QIU AND B. ZHU, Random and interval uncertain symplectic methods for linear Birkhoff equations and their comparison study, Scientia Sinica Phys. Mech. Astron., 50(8) (2020), 084611 (in Chinese).

[52] H. L. SU, Birkhoffian symplectic scheme for a Quantum system, Commun. Theor. Phys., 53 (2010), pp. 476-480. 